\title{
Impact of early postbariatric surgery acute kidney injury on long-term renal function
}

\begin{abstract}
Background: Bariatric surgery can improve renal dysfunction associated with obesity and diabetes. However, acute kidney injury (AKI) can complicate the early postoperative course after bariatric surgery. The long-term consequences of early postoperative AKI on renal function are unknown. Methods: Patient undergoing bariatric surgery from 2008 to 2015 who developed AKI within 60 days after surgery were studied. Patients on dialysis before surgery were excluded. Results: Out of 4722 patients, 42 patients (0.9\%) developed early postoperative AKI after bariatric surgery of whom five had chronic kidney disease (CKD) preoperatively including CKD stage $3(n=2)$, stage $4(n=2)$, and stage $5(n=1)$. Etiologies of AKI included prerenal in 37 and renal in 5 patients. Nine patients $(21 \%)$ underwent hemodialysis in early postoperative period for AKI. The median duration of follow-up was 28 months (interquartile range, 4-59). Of the 40 patients eligible for follow-up, 36 patients $(90 \%)$ returned to their baseline renal function. However, four patients $(10 \%)$ had worsening of renal function at follow-up. Conclusions: The incidence of early postoperative AKI after bariatric surgery is about $1 \%$. The most common causes of AKI after bariatric surgery are dehydration and infectious complications. In our series, $10 \%$ of patients who developed AKI in early postoperative period had worsening of renal function in long-term follow-up. In the absence of severe sepsis and severe underlying kidney dysfunction (CKD stages 4 and 5), full recovery is expected after postoperative AKI.
\end{abstract}

Keyword: Acute kidney injury; Bariatric surgery; Obesity; Sleeve gastrectomy; Gastric bypass; Complication; Renal failure 